\title{
An Improved Uneven Multilayer Cluster Routing Algorithm for Homogeneous WSNs Based on Cooperative Relaying
}

\author{
Siran WANG $^{1}$, Yanxia WANG ${ }^{2, a}$ and Yunjian TANG ${ }^{3, b}$ \\ ${ }^{1,2,3}$ Chongqing Academy of Science and Technology,Chongqing,401123,China \\ a260789093@qq.com, bcqu_tyj@126.com
}

\begin{abstract}
Keywords: Wireless sensor network (WSN), Cluster routing, Cluster, Relay node, Energy consumption.

Abstract: Aiming to alleviate the drawbacks of the existing power-aware routing algorithms caused by the hotspot problem and Near-Far problem, this paper propose an improved uneven clustering routing algorithm , named as Multilayer Cluster Routing based on Cooperative Relaying (MCRCR). This new algorithm protocol introduces the super-cluster-head node and the relay node conceptions to avoid the hotspots and balance the node energy consumption. After a series of mathematical calculations, the energy consumption for each type of sensor node is provided. Based on MATLAB simulation results, a conclusion can be shown that the MCRCR algorithm is significantly effective in extending the operation lifetime of homogenous WSNs.
\end{abstract}

\section{I . Introduction}

Since sensors can be located in some inhospitable terrains like the thousands of square kilometers of rivers and lacks, it is promising that WSNs can be widespread applied in the monitoring and detection of hydrographic environment. Thanks to the tough positions of the sensors, it is infeasible to recharge the sensor power [1]. The low battery capacity of wireless sensor node poses a chief challenge on applications of WSNs[2]. [3] illustrates that the increasing number of died sensor nodes witness a remarkably sharp downward trend in capability of the network. A recommended WSN is efficient in conserving energy.

Many hierarchical routing algorithm protocols have been presented during the past decades. The low-energy adaptive clustering hierarchy (LEACH) [4], as a typical protocol architecture for WSN, is proposed by Heinzelman. The obvious deficiency of LEACH is that the cluster-head $(\mathrm{CH})$ is selected in a random manner without the consideration of residual energy. It exerts a deep restriction in extending the lifetime of the WSNs. LEACH-centralized (LEACH-C) [5], LEACH-fixed (LEACH-F) and Deterministic Cluster-Head Selection (DCHS) [6] which use the LEACH as a basis for improving the energy efficiency are invented quickly. In [7], Lindsey demonstrates PEGASIS (Power-Efficient Gathering in Sensor Information Systems) based on the chain structure might provide double lifetime of the WSN system, compared with LEACH. Manjeshwar and P.Agrawal excogitate a neoteric protocol, named TEEN (Threshold Sensitive Energy Efficient Sensor Network) [8], to enhanced efficiency by setting the hard threshold and soft threshold in advance. Choi works into this field in the aspects of the two-phase clustering scheme [9] and the coverage-data reporting latency tradeoff [10], respectively.

In this paper, a new algorithm protocol named Multilayer Cluster Routing based on Cooperative Relaying (MCRCR) is proposed to tackle the hotspot problem and Near-Far problem which existing routing protocols do not consider. MCRCR algorithm protocol combines the benefits of hierarchical routing and cooperative relaying routing to improving the energy efficiency of WSNs.

The residual of this paper is organized as follows. In Section II, details about the MCRCR algorithm protocol are discussed. Section III describes the system model and energy consumption model of the MCRCR, respectively. Section IV presents the performance comparison of MCRCR with the LEACH via simulations. Finally, Section V concludes the paper. 


\section{II . MCRCR Algorithm}

This paper divides MCRCR algorithm into three phases: Cluster-Head Communication Phase, Super-Cluster-Head Communication Phase and Stable Data Transmission Phase. The algorithm details are described in the subsections.

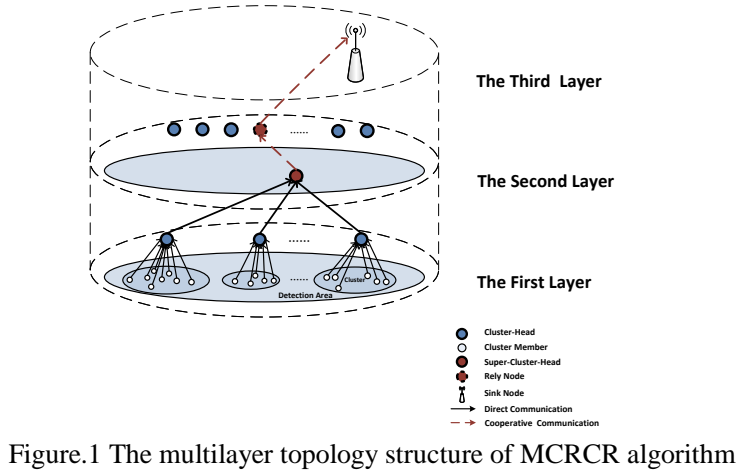

\section{A. Phase I : Cluster-Head Communication Phase}

In Figure.1, there is one sink node and N-number sensor nodes in the S square meters application scope. As to each cluster, there is only one sensor acts as cluster-head $(\mathrm{CH})$, and the other sensors belong to cluster member (CM). Referencing to the LEACH and EEUC (Energy Efficient Uneven Clustering) [11], MCRCR defined that CHs are selected in each round. Moreover, CM nodes send information to $\mathrm{CH}$ in direct link because of the relatively small acreage of each cluster. The subsequent administrative operations like data compression and retransmission are computed in $\mathrm{CH}$.

At first, the sink node broadcasts one initialization massage. Each sensor node listens it and estimates the distance from sink node based on RSSI (received signal strength indicator), denoted as $\mathrm{d}(\mathrm{i}$,sink). Then at the beginning of each round, every alive sensor node broadcasts a data packet within a radius $\mathrm{R}_{\text {ad }}$ firstly, which contains the ID, geographic location $\mathrm{d}(\mathrm{i}$,sink) and residual energy $\mathrm{E}_{\mathrm{re}}$. Hence each node is aware of the position and residual energy about its adjacent nodes in every round. As to the current round, the node which is not the $\mathrm{CH}$ in the previous round should produce an arbitrary number between 0 and 1 randomly. If the number exceed the threshold $\mathrm{T}(\mathrm{n})$, and the node does not receive any an advertisement (ADV) message. Then the node becomes the candidate for $\mathrm{CH}$ and broadcast $\mathrm{ADV}$ within a radius $\mathrm{R}_{\mathrm{CH}}$. If the received nodes had not added to any cluster, they response a JION message to this $\mathrm{CH}$ and become one of the $\mathrm{CM}$ for this cluster. Once the sensor node acts as a CM node, it will goes to sleep model and does not communicate with other clusters. Wait until the sink node has selected the super-cluster-head ( $\mathrm{SCH}$ ) and the relay nodes before the sink node activates the whole CMs.

$$
T(n)=\left\{\begin{array}{cl}
\frac{p}{1-p\left(r \times \bmod \left(\frac{1}{p}\right)\right)}, & \text { if } n \in G \\
0, & \text { otherwise }
\end{array}\right.
$$

where $\mathrm{p}$ is the desired percentage of being a $\mathrm{CH}, \mathrm{r}$ illustrates the round number and $\mathrm{G}$ presents the set that the nodes which are not selected to be the $\mathrm{CH}$ during the $1 / \mathrm{p}$ of the last rounds. If the residual energy below the energy threshold $E_{\text {min }}$, this node declares to add the died node set $\mathrm{D}$, and send a DEAD message to $\mathrm{CH}$. Thus the number of died node is known by WSN. Furthermore, the alive node density $\eta$, can be estimated in each round.

$$
\eta=\frac{\mathrm{N}_{\text {alive }}}{\mathrm{N}} \text {, }
$$


where $\mathrm{N}_{\text {alive }}$ and $\mathrm{N}$ are the number of alive nodes in the current round and the initial number of nodes, respectively. Defined the competitive radius of the $\mathrm{CH}$ as $\mathrm{R}_{\mathrm{CH}}$. Referencing to [13], the competitive radius in this paper is formulated as:

$$
\mathrm{R}_{\mathrm{CH}}=\mathrm{R}_{\mathrm{ad}}\left(1-\mathrm{C}_{\mathrm{R}} \frac{\mathrm{d}_{\text {max }}-\mathrm{d}(\mathrm{i}, \mathrm{sink})}{\mathrm{d}_{\max }-\mathrm{d}_{\min }} \times \frac{\mathrm{E}_{0}-\mathrm{E}_{\mathrm{re}}}{\mathrm{E}_{\mathrm{re}}-\mathrm{E}_{\min }}\right)\left(1-\left(1-\mathrm{C}_{\mathrm{R}}\right) \eta\right) \text {, }
$$

where $R_{a d}$ is the pre-set maximum of the competitive radius; $C_{R}$ is a constant for controlling the value range, $C_{R} \in(0,1]$; the $d_{\max }$ and $d_{\text {min }}$ represent maximum value and minimum value of the node i-to-sink node distance, respectively. $\mathrm{E}_{0}$ is the initial energy of each node.

\section{B. Phase II : Super-Cluster-Head Communication Phase}

In Phase II, data packets are delivered from CHs to the unique SCH firstly, and then data re-transmit to sink node like the base station (BS) in cooperative communication link. The topology of the super-cluster-head communication phase is modified and evolved from the topology of BCDCP (Base-Station Controlled Dynamic Clustering protocol) [14]. The SCH consumes more power than the other sensors. In order to delay the death time, WSN updates $\mathrm{SCH}$ in every round. In this phase, each $\mathrm{CH}$ calculates it own weight firstly, and then send data to sink node. The sink node ranks the weights of $\mathrm{CHs}$ in descending order. The $\mathrm{CH}$ with the largest weight evolves into the SCH. Denotes $\mathrm{W}_{\mathrm{i}}$ as the individual weight of $\mathrm{CH}$ :

$$
\mathrm{W}_{\mathrm{i}}=\frac{\mathrm{C}_{\mathrm{w}} \mathrm{E}_{\mathrm{re}}}{\mathrm{E}_{0}}+\frac{\left(1-\mathrm{C}_{\mathrm{w}}\right) \eta \mathrm{M}_{\mathrm{CH}}}{\mathrm{N}} \quad \text { with } \mathrm{M}_{\mathrm{CH}}=\sqrt{\frac{\mathrm{N}}{2 \pi} \times \frac{\alpha_{\mathrm{fs}}}{\alpha_{\mathrm{mp}}} \times \frac{\mathrm{s}}{\mathrm{d}(\mathrm{i}, \operatorname{sink})^{2}}} \text { [5], }
$$

where $\mathrm{M}_{\mathrm{CH}}$ is the optimal number of CHs; $\alpha_{\mathrm{fs}}$ and $\alpha_{\mathrm{mp}}$ are energy consumption factor of the amplifier circuit in free space propagation model and multi-path fading model respectively; $\mathrm{C}_{\mathrm{w}}$ is a constant, $\mathrm{C}_{\mathrm{W}} \in(0,1]$. SCH selection of MCRCR relies on the residual energy and the distance to the sink node. The high-power nodes and near-BS nodes got more opportunity to be SCH.

In [15], Murugunathan proposed that BCDCP transmits data from $\mathrm{CH}$ to $\mathrm{BS}$ in a direct transmission manner. It is not suitable for a long-distance transmission. MCRCR algorithm protocol, however, combines the cooperative relaying technology to forward data from SCH to sink node. It is more energy-efficient than BCDCP because of compensation for the signal loss and improvement for the quality of service. The cooperative relaying selection stage based on relaying programs are required to add into Phase II . Defined that MCRCR allows sink node selects one of the CHs to act as the relay node in Phase II, if it satisfies the following two equations. Otherwise, the SCH transfer messages to sink node directly.

$$
\begin{aligned}
& \mathrm{d}_{\mathrm{S}, \text { sink }}{ }^{2} \geq \mathrm{d}_{\mathrm{S}, \mathrm{R}}{ }^{2}+\mathrm{d}_{\mathrm{R}, \text { sink }}{ }^{2} ; \\
& \mathrm{d}_{\mathrm{R}, \text { sink }}=\min \left(\mathrm{d}_{\mathrm{S}, \text { sink }}, \mathrm{d}_{\mathrm{CH}, \text { sink }}\right) .
\end{aligned}
$$

where $d_{C H \text {,sink }}$ presents the distance between $\mathrm{CH}$ and the sink node. $d_{\mathrm{S} \text {,sink }}$ and $\mathrm{d}_{\mathrm{R} \text {,sink }}$ represent the distance between SCH and the sink node and distance between the relay node and the sink node, respectively.

\section{Phase III: Stable Data Transmission Phase}

The MCRCR protocol accesses to the intra-cluster data transmission stage firstly. In [5], [16], [17], authors illustrated that TDMA/CDMA (Time Division Multiple Access/ Code Division Multiple Access) is a superior protocol for collision avoidance and energy compensation. The $\mathrm{CH}$ creates a TDMA schedule to guarantee data transmitting from the each $\mathrm{CM}$ node to $\mathrm{CH}$ in an assigned timeslot. In order to avoid Radio interference caused by transmitting data among the multiple-access channel in 
form of a distributed manner, all nodes adopt the CDMA code for data transmission [4], [7]. As to the inter-cluster data transmission stage, $\mathrm{SCH}, \mathrm{CHs}$ and relay node also employ the TDMA/CDMA to transfer data.

\section{System Model and Energy Consumption Model}

We employ the homogenous WSN with N-number static sensors in this paper. Assuming that sensors have the same initial energy and unique ID number and sit in a $\mathrm{L} \times \mathrm{L}$ scope in forms of the randomly distribution. Communication links are symmetric so that the forward and reverse channels keep constant in the period of each round. Each sensor shares the information with the others through different MAC protocols. BS locates in a fixed position and keeps far distance from the WSN systems.

This paper uses the same energy consumption model as [4], [5], and the energy consumption of the transmit node and receive node for sending a k-bit message in d-meter distance are given by the Equation.(7) and Equation.(8), respectively:

$$
\begin{aligned}
& \mathrm{E}_{\mathrm{Tx}}(\mathrm{k}, \mathrm{d})=\mathrm{kE}_{\text {elec }}+\mathrm{k} \propto \mathrm{d}^{\lambda}=\left\{\begin{array}{l}
\mathrm{kE}_{\text {elec }}+\mathrm{k}_{\mathrm{fs}} \mathrm{d}^{2}, \mathrm{~d}<\mathrm{d}_{\text {th }} \\
\mathrm{kE}_{\text {elec }}+\mathrm{k}_{\mathrm{mp}} \mathrm{d}^{4}, \mathrm{~d}>\mathrm{d}_{\text {th }}
\end{array} \quad \text { with } \mathrm{d}_{\mathrm{th}}=\sqrt{\frac{\alpha_{\mathrm{fs}}}{\alpha_{\mathrm{mp}}}} ;\right. \\
& \mathrm{E}_{\mathrm{Rx}}(\mathrm{k})=\mathrm{kE}_{\text {elec }} ; \\
& \mathrm{E}_{\mathrm{a}}=\mathrm{kE}_{\text {data }} ;
\end{aligned}
$$

where $E_{\text {elec }}$ denotes the electronic energy consumption for data processing ( in J/bit). $\propto$ is the energy consumption factor. $\lambda$ is the exponent coefficient, which is relies on desirable the bit-error rate. $d_{\text {th }}$ is the threshold for the node-to-node distance $d$ [18], [19]. $E_{a}$ is the energy cost function for data aggregation of $\mathrm{CH}$, and $\mathrm{E}_{\text {data }}$ is the energy for aggregating one bit data.

The sum energy consumption of $\mathrm{CM}$, denoted as $\mathrm{E}_{\mathrm{CM}}$, is formulated as:

$$
\mathrm{E}_{\mathrm{CM}}=\mathrm{E}_{\mathrm{Tx}}\left(\mathrm{k}, \mathrm{d}_{\mathrm{n}_{\mathrm{i}}, \mathrm{CH}}\right)=\mathrm{kE}_{\text {elec }}+\mathrm{k} \propto \frac{1}{2 \pi} \times \frac{\mathrm{L}^{2}}{\mathrm{M}_{\mathrm{CH}}} \text {. }
$$

Taking the three phases into consideration, the sum energy consumption of the ordinary $\mathrm{CH}$, the relay node and $\mathrm{SCH}$ are:

$$
\begin{aligned}
& \mathrm{E}_{\mathrm{CH}}=\left(\frac{N}{\mathrm{M}_{\mathrm{CH}}}-1\right) \mathrm{E}_{\mathrm{Rx}}(\mathrm{k})+\frac{\mathrm{N}}{\mathrm{M}_{\mathrm{CH}}} \mathrm{E}_{\mathrm{a}}+\mathrm{E}_{\mathrm{Tx}}\left(\mathrm{k}, \mathrm{d}_{\mathrm{CH}, \mathrm{S}}\right) ; \\
& \mathrm{E}_{\mathrm{CH}}^{\text {relay }}=\frac{\mathrm{N}}{\mathrm{M}_{\mathrm{CH}}} \mathrm{E}_{\mathrm{Rx}}(\mathrm{k})+\frac{\mathrm{N}}{\mathrm{M}_{\mathrm{CH}}} \mathrm{E}_{\mathrm{a}}+\mathrm{E}_{\mathrm{Tx}}\left(\mathrm{k}, \mathrm{d}_{\mathrm{R}, \text { sink }}\right) ; \\
& \mathrm{E}_{\mathrm{SH}}=\frac{\mathrm{N}+\mathrm{M}_{\mathrm{CH}}{ }^{2}-2 \mathrm{M}_{\mathrm{CH}}}{\mathrm{M}_{\mathrm{CH}}} \mathrm{E}_{\mathrm{Rx}}(\mathrm{k})+\frac{\mathrm{N}}{\mathrm{M}_{\mathrm{CH}}} \mathrm{E}_{\mathrm{a}}+\mathrm{E}_{\mathrm{Tx}}\left(\mathrm{k}, \mathrm{d}_{\mathrm{S}, \mathrm{R}}\right) ;
\end{aligned}
$$

where $\mathrm{d}_{\mathrm{CH}, \mathrm{S}}$ is the distance between $\mathrm{CH}$ and $\mathrm{SCH}$.

In addition, the WSN system consumes the gross energy for each round in MCRCR can be calculated as:

$$
\mathrm{E}_{\mathrm{G}}=(\mathrm{N}-\mathrm{M}) \mathrm{E}_{\mathrm{CM}}+(\mathrm{M}-2) \mathrm{E}_{\mathrm{CH}}+\mathrm{E}_{\mathrm{CH}}^{\text {relay }}+\mathrm{E}_{\mathrm{SH}} \text {. }
$$

\section{Simulation Results}

TABLE.1 CONFIGURATION of THE SIMULATION ENVIRONMENT

\begin{tabular}{|l|l|}
\hline System Parameter & Value \\
\hline Simulation Area & 200 meters $\times 200$ meters \\
\hline Sink Node (BS) Location & $(400,100) \mathrm{m}$ \\
\hline Number of Nodes & 100 \\
\hline
\end{tabular}




\begin{tabular}{|c|c|}
\hline Data Packet Size & 4000 bits \\
\hline Initial Energy & $0.5 \mathrm{~J}$ \\
\hline$E_{\text {glas }}$ & $50 \mathrm{~nJ} / \mathrm{bit}$ \\
\hline$E_{\text {dxtz }}$ & $5 \mathrm{~nJ} / \mathrm{bit} / \mathrm{signal}$ \\
\hline$E_{\min }$ & $0.025 \mathrm{~J}$ \\
\hline$\sigma_{f_{2}}$ & $10 \mathrm{pJ} / \mathrm{bit} / \mathrm{m}^{2}$ \\
\hline$a_{m p}$ & $0.0013 \mathrm{pJ} / \mathrm{bit} / \mathrm{m}^{4}$ \\
\hline$p$ & $5 \%$ \\
\hline$R_{x d}$ & $50 \mathrm{~m}$ \\
\hline
\end{tabular}

Section IV aims to demonstrate the performance of MCRCR algorithm protocol by making a comparison with LEACH through MATLAB simulation. Table.1 presents the configuration of the simulation environment.
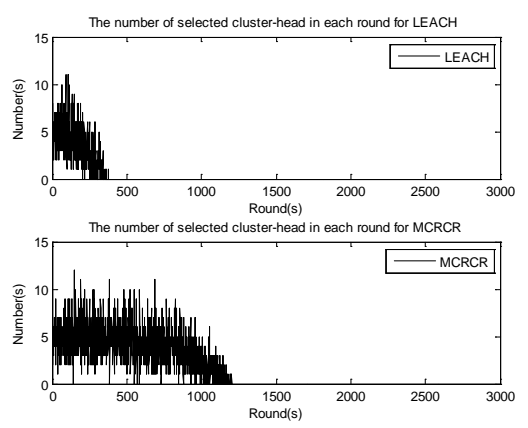

Figure.2 The numbers of selected cluster-head in each round.

The optimal number of CHs has been formulated as the Equation.(19) in [5]. It is computed that for a 100-sensor node WSN, choosing 5 CHs achieves the optimal performance. In Figure.2, the number of CHs fluctuates around the 5. As a consequence, the simulation result can support Heinzelman's research.

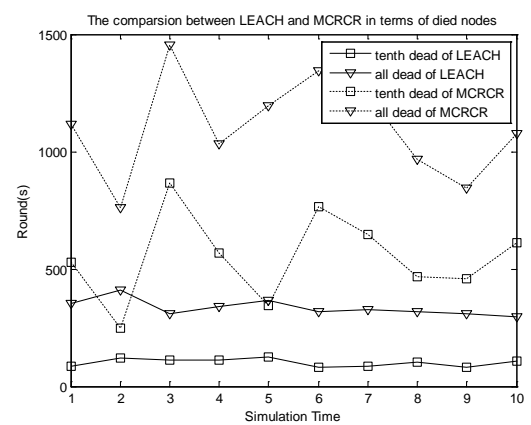

Figure.3 The comparison between LEACH and MCRCR in terms of died nodes.

MATLAB has run ten simulations for three thousand rounds to demonstrate the data packets transmission performance of the proposed MCRCR algorithm protocol. It has been shown in Figure.3 that compared with LEACH, MCRCR shows a great improvement in the lifetime of the WSN. In MCRCER, the situations that one in ten sensor nodes die and all sensor nodes die are approximately appeared in the Round 551 and Round 1100, respectively. As to the LEACH, they might happen in the Round 102 and the Round 335, respectively. Hence, the operation time of this new proposed algorithm increases more than double. 

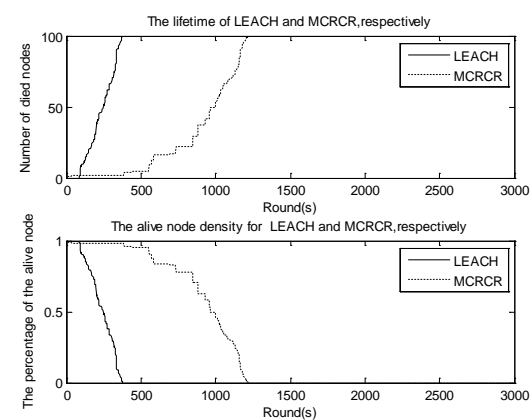

Figure.4 The lifetime and alive node density of LEACH and MCRCR, respectively.

As the Section II mentioned above, MCRCR combines the multi-hop relaying transmission model and uneven cluster formation to tackle the Near-Far Problem and the hotspot Problem. Figure.4 illustrates that under the same configuration of the simulation environment when all the nodes which adopt LEACH run out of power, MCRCR can ensure that the alive node density still keep in a high level. Since MCRCR provides a better cluster-head selection mechanism to balance the energy consumption of each sensor.
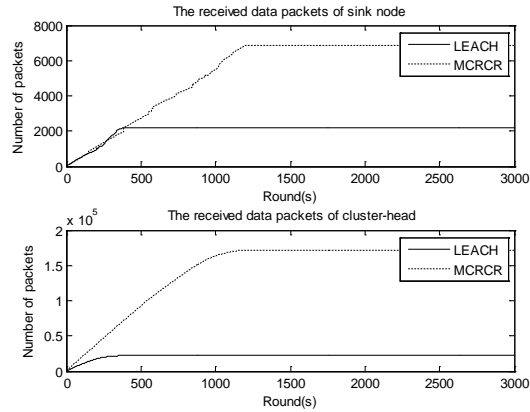

Figure.5 The sink node receives data packets based on LEACH and MCRCR, respectively.

The number of received data packets increases along with the growth of lifetime of the WSN, as the Figure. 5 shown. No matter the sink node or the cluster-head, MCRCR shows the great effectiveness in receiving data. For the sink node, MCRCR obtains more than three times as many data packets as LEACH. As to the CHs, the number of received data packets of MCRCR is six times over that of LEACH.

\section{Conclusion}

This paper proposes MCRCR as a new clustering routing algorithm protocol for a long-distance transmission WSN. It consists of three phase: Cluster-Head Communication Phase, Super-Cluster-Head Communication Phase and Stable Data Transmission Phase. In order to avoid the near-BS nodes becoming the hotspots, the $\mathrm{CH}$ selection scheme introduces the competitive radius conception to constructed uneven cluster. The number of cluster rise along with the decrease of the node-to-sink node distance. The SCH and relay node based on the multi-hop data transmission prolong the lifetime further. WSN uses TDMA/CDMA protocol to delivery data. Comparing the transmission distance with the threshold, each node can estimate the transmit energy consumption. The MATLAB simulation results indicate that the MCRCR algorithm protocol improves the balance of WSN energy consumption, postpones the birth of died nodes and prolongs the lifetime of the WSN significantly.

\section{Acknowledgements}

This work was supported by 2014 National Project for Subsidy Funds of the Development of Internet of Things (the contract number: 2014083101). 


\section{References}

[1] G. Lim and L. J. Cimini, "Energy-Efficient Cooperative Beamforming in Clustered Wireless Networks,” vol. 12, no. 3, pp. 1376-1385, 2013.

[2] A. E. A. A. Abdulla, H. Nishiyama, and N. Kato, "Extending the lifetime of wireless sensor networks : A hybrid routing algorithm," Comput. Commun., vol. 35, no. 9, pp. 1056-1063, 2012.

[3] A. Liu, J. Ren, X. Li, Z. Chen, and X. Sherman, "Design principles and improvement of cost function based energy aware routing algorithms for wireless sensor networks,” vol. 56, pp. 1951-1967, 2012.

[4] W. R. Heinzelman, A. Chandrakasan, and H. Balakrishnan, "Energy-Efficient Communication Protocol for Wireless Microsensor Networks,” vol. 00, no. c, pp. 1-10, 2000.

[5] W. B. Heinzelman, A. P. Chandrakasan, S. Member, and H. Balakrishnan, "An Application-Specific Protocol Architecture for Wireless Microsensor Networks,” vol. 1, no. 4, pp. 660-670, 2002.

[6] C. Science, "Low Energy Adaptive Clustering Hierarchy with Deterministic Cluster-Head Selection,” pp. 368-372.

[7] S. Lindsey, C. Raghavendra, F. Member, K. M. Sivalingam, and S. Member, "Data Gathering Algorithms in Sensor Networks Using Energy Metrics,” vol. 13, no. 9, pp. 924-935, 2002.

[8] A. Manjeshwar and D. P. Agrawal, "TEEN : A Routing Protocol for Enhanced Efficiency in Wireless Sensor Networks,” vol. 00, no. C, 2001.

[9] W. Choi, P. Shah, and S. K. Das, "A Framework for Energy-Saving Data Gathering Using Two-Phase Clustering in Wireless Sensor Networks,” 2004.

[10] W. Choi and S. K. Das, "Trade-off Between Coverage and Data Reporting Latency for Energy-Conserving Data Gathering in Wireless Sensor Networks,” pp. 503-512, 2004.

[11] Chengfa.Li,Guihai.Chen,Mao.Ye,Jie.Wu,“An Uneven Cluster Based Routing Protocol for Wireless Sensor Networks”, pp.27-36,2007

[12] S. R. Heikalabad and A. H. Navin, "REACH : The New Routing Algorithm based on Energy Aware Clustering Hierarchical for Lifetime Increasing in Wireless Sensor Networks,” vol. 2, no. Iceie, pp. 288-291, 2010.

[13] Ran.Yan,Yun.Yang,Tingjun.Shi,Xiuping.Kong,Wenchun.Xu, “Uneven Cluster Routing Algorithm Based on Node Location and Node Desnity.”,Vol.42,No.8,Aug,2015.

[14] X. Liu, “ A Survey on Clustering Routing Protocols in Wireless Sensor Networks. ”2012.

[15] Murugunathan, S.D.; Ma, D.C.F.; Bhasin, R.I.; Fapajuwo, A.O. A Centralized Energy-Efficient Routing Protocol for Wireless Sensor Networks. IEEE Radio Commun. 2005, 43, S8-S13.

[16] F. Salvi-garau and M. Stojanovic, "Multi-Cluster Protocol for Ad Hoc Mobile Underwater Acoustic Networks,” pp. 91-98.

[17] M. C. Domingo and R. Prior, "A DISTRIBUTED CLUSTERING SCHEME FOR UNDERWATER WIRELESS SENSOR NETWORKS,” 2007.

[18] I. K. Samaras, G. D. Hassapis, S. Member, and J. V Gialelis, “A Modified DPWS Protocol Stack for 6LoWPAN-Based Wireless Sensor Networks,” vol. 9, no. 1, pp. 209-217, 2013.

[19] D. Zhang and Y. Liang, "A kind of novel method of service-aware computing for uncertain mobile applications,” Math. Comput. Model., vol. 57, no. 3-4, pp. 344-356, 2013. 